\title{
Chemical constituents of Chirita drakei Burtt collected in Ha Long Bay, Quang Ninh province, Viet Nam
}

\section{Part 2. Compounds isolated from the $n$-butanol extract}

\author{
Nguyen Thi Hoang Anh ${ }^{1,2}$, Nguyen Van Tuan ${ }^{2,3}$, Tran Duc Quan ${ }^{1}$, Dao Duc Thien ${ }^{1}$, \\ Nguyen Thanh Tam ${ }^{1}$, Pham Duc Thang ${ }^{4}$, Trinh Thi Thuy ${ }^{1}$, Tran Van Sung ${ }^{1}$ \\ ${ }^{1}$ Institute of Chemistry, Vietnam Academy of Science and Technology \\ ${ }^{2}$ Graduate University of Science and Technology, Vietnam Academy of Science and Technology \\ ${ }^{3}$ Asean College, Van Lam, Hung Yen \\ ${ }^{4}$ Danang University of Medical Technology and Pharmacy
}

Received 14 March 2017; Accepted for publication 28 August 2017

\begin{abstract}
Four phenol glycosides together with cannabiside B were isolated from the $n$-butanol extract of the aerial part of Chirita drakei Burtt collected in islands, on mountain slopes of Ha Long bay, Quang Ninh province. Their structures have been elucidated by mass, NMR spectroscopy and comparison with published data. Compounds $\mathbf{4}$ and $\mathbf{5}$ were obtained for the first time from the genera Chirita.
\end{abstract}

Keywords. Chirita drakei, phenylethanoid glycoside, phenol glucoside, $\alpha, \beta$-unsaturated $\gamma$-lactone C-glucoside.

\section{INTRODUCTION}

In a previous article [1] we have reported the isolation and structure determination of compounds with the medium polarity from $n$-hexane and ethyl acetate extracts of aerial parts of Chirita drakei Burtt plant collected in the islands, limestone slopes in the Ha Long Bay, Quang Ninh province. This paper reports continuously the isolation and structure elucidation of the more polar components from $n$ butanol extract, including three phenylethanoid glycosides (1-3), one phenol glucoside (4) and an $\alpha, \beta$-unsaturated $\gamma$-lactone C-glucoside (Cannabiside B) (5).

\section{EXPERIMENTAL}

\subsection{Equipments and methods}

IR: Impact 410, Nicolet, Germany; ESI-MS: LCMSD-Trap-SL, Varian, USA, NMR: Bruker Avance 500, Germany with TMS as internal reference (for ${ }^{1} \mathrm{H}$ ) and solvent signal (for ${ }^{13} \mathrm{C}$ ). $\mathrm{CC}$ used silica gel 60G, size 0.043-0.063 mm (Merck), TLC: precoated silica gel G60F254 plates (Merck), spots were detected by spraying with vanillin $1 \%$ in conc. $\mathrm{H}_{2} \mathrm{SO}_{4}$ and heating at $110{ }^{\circ} \mathrm{C}$.

\subsection{Plant material}

The aerial parts of $C$. drakei were collected in the islands of Ha Long Bay, Quang Ninh province, Vietnam in October 2013. A voucher specimen (VHH.HL 10.2013.1) is deposited in Institute of Chemistry, VAST, Hanoi, Vietnam. The scientific name was identified by Dr. Tran Thi Phuong Anh, Vietnam National Museum of Nature, VAST, Hanoi, Vietnam.

\subsection{Extraction and isolation}

The dried powdered aerial parts of $C$. drakei $(1.7 \mathrm{~kg})$ were extracted exhaustively with $\mathrm{MeOH}: \mathrm{H}_{2} \mathrm{O}$ (9:1) at room temperature. The methanol extract was concentrated under vacuum and then aq. solution. was extracted with $n$-hexane, EtOAc and $n-\mathrm{BuOH}$, successively. The solvent was evaporated in vacuum to afford $n$-hexane $(10.0 \mathrm{~g})$, EtOAc $(10.8 \mathrm{~g})$ and $n$ $\mathrm{BuOH}(55.0 \mathrm{~g})$ extracts. The $n$ - $\mathrm{BuOH}$ extract $(55 \mathrm{~g})$ was chromatographed on a silica gel column, eluted with a gradient solvent system of $\mathrm{CH}_{2} \mathrm{Cl}_{2}: \mathrm{MeOH}$ (from 100:0 to 0:100), to give 5 fractions (F1-F5). The fraction F4 (11 g) was subjected to silica gel column, eluting with a gradient system of EtOAc: $\mathrm{MeOH}: \mathrm{H}_{2} \mathrm{O}$ (from 5:0.5:0.1 to 2.5:0.5:0.1), 
followed by RP-18 (MeOH: $\left.\mathrm{H}_{2} \mathrm{O}, 2: 3\right)$ and Sephadex LH-20 (MeOH) to yield compounds $1(20 \mathrm{mg}), 2$ $(10 \mathrm{mg}), \mathbf{4}(10 \mathrm{mg})$ and $5(10 \mathrm{mg})$. The fraction F5 (7 g) was purified over column silica gel, eluting with a gradient system of $\mathrm{CH}_{2} \mathrm{Cl}_{2}: \mathrm{MeOH}: \mathrm{H}_{2} \mathrm{O}$ (from 4:1:0.1 to 2:1:0.1) followed by RP-18 (aceton: $\mathrm{H}_{2} \mathrm{O}$, 3:7) and Sephadex LH-20 (MeOH) to yield 3 (10 $\mathrm{mg})$.

\section{RESULTS AND DISCUSSION}

The structures of five compounds isolated from $n$ $\mathrm{BuOH}$ extract of $C$. drakei were identified as 2-(3,4dihydroxyphenyl)ethyl- $\beta$-D-glucopyranoside desrhamnosyl isoacteoside (2), chiritoside C (3), brachyanin D (4) and cannabiside B (5) by the analysis of their 1D, 2D-NMR and ESI-MS spectra and comparison with published data.

Compound 1, obtained as amorphous pale yellow powder. It has the pseudo-molecular ion peak at $m / z 315[\mathrm{M}-\mathrm{H}]^{-}$in the negative ESI-MS spectrum, according to the molecular formula $\mathrm{C}_{14} \mathrm{H}_{20} \mathrm{O}_{8}$. Its ${ }^{1} \mathrm{H}$ and ${ }^{13} \mathrm{C}$-NMR spectra revealed the signals of a 1,3,4trisubstituted phenylethyl alcohol: three aromatic protons at $\delta_{\mathrm{H}} 6.71(\mathrm{~d}, J=1.5 \mathrm{~Hz}) ; 6.69(\mathrm{~d}, J=8.5$ $\mathrm{Hz}) ; 6.57$ (dd, $J=1.5 \& 8.5 \mathrm{~Hz}$ ), oxymethylene protons at $\delta_{\mathrm{H}} 3.72(\mathrm{~m}), 4.05(\mathrm{~m})$, methylene protons at $\delta_{\mathrm{H}} 2.80(\mathrm{~m})$ and one $\beta$-glucopyranose unit: anomeric signals at $\delta_{\mathrm{H}} 4.31(\mathrm{~d}, J=8.0 \mathrm{~Hz})$ and $\delta_{\mathrm{C}}$ 104.35. The spectroscopic data of 1 were identical with those of 2-(3,4-dihydroxyphenyl)ethyl- $\beta$-Dglucopyranoside in the literature [2].

Compound 2, obtained as a pale yellow powder. The molecular formula of $\mathbf{2}$ was determined to be $\mathrm{C}_{23} \mathrm{H}_{26} \mathrm{O}_{11}$, based on the positive ESI-MS $(\mathrm{m} / z 501$ $\left.[\mathrm{M}+\mathrm{Na}]^{+}\right)$and NMR data. The ${ }^{1} \mathrm{H}$ and ${ }^{13} \mathrm{C}-\mathrm{NMR}$ spectra of $\mathbf{2}$ were very similar to those of $\mathbf{1}$ but appeared additionally the signals of a trans-caffeoyl moiety with an $\mathrm{ABX}$ spin system at $\delta_{\mathrm{H}} 7.05(\mathrm{~d}, J=$ $1.5 \mathrm{~Hz}), 6.78(\mathrm{~d}, J=8.0 \mathrm{~Hz}), 6.91(\mathrm{dd}, J=1.5 \& 8.0$ $\mathrm{Hz})$; a trans double bond at $\delta_{\mathrm{H}} 6.30(\mathrm{~d}, J=15.7)$, $7.58(\mathrm{~d}, J=15.7 \mathrm{~Hz})$ and a carbonyl carbon at $\delta_{\mathrm{C}}$ 169.29. The connection of the caffeoyl moiety in 2 was determined at C-6' of $\beta$-glucopyranose by the downfield shifted signals of $\mathrm{CH}_{2}-6$ compared with those of $1 \quad\left(\Delta \delta_{\mathrm{C}}=1.92\right.$ and $\left.\Delta \delta_{\mathrm{H}}=0.65\right)$. Consequently, the structure of $\mathbf{2}$ was determined to be desrhamnosyl isoacteoside by comparison with reported data [3].

Compound 3, pale yellow powder, showed pseudo-molecular ion peak at $\mathrm{m} / z 639[\mathrm{M}-\mathrm{H}]^{-}$ according to $\mathrm{C}_{29} \mathrm{H}_{36} \mathrm{O}_{16}$. Its NMR data indicated that 3 is also a phenylethanoid glycoside with phenylethyl alcohol, caffeoyl and two glucopyranose units. The two anomeric protons at $\delta_{\mathrm{H}} 4.88(\mathrm{~d}, J=$ $7.5 \mathrm{~Hz})$ and $4.35(\mathrm{~d}, J=8.0 \mathrm{~Hz})$ confirmed this suggestion. The connection of the second $\beta$ glucopyranosyl unit at C-4' of caffeoyl group was identified when compared with spectroscopic data of chiritoside $\mathrm{C}$ in [4]. Chiritoside $\mathrm{C}$ was one of the first three representatives possessed $\beta$ glucopyranosyl moiety at C-4 of caffeoyl group, isolated from Chirita sinensis since 1994.

Compound $\mathbf{4}$ was obtained as a colourless oil. Its molecular formula was determined to be $\mathrm{C}_{20} \mathrm{H}_{28} \mathrm{O}_{11}$ based on the NMR and positive ESI-MS data $(\mathrm{m} / \mathrm{z}$ $\left.467[\mathrm{M}+\mathrm{Na}]^{+}\right)$. Its ${ }^{1} \mathrm{H}-\mathrm{NMR}$ spectrum showed three aromatic $\mathrm{ABX}$ protons at $\delta_{\mathrm{H}} 6.97(\mathrm{~d}, J=2.0 \mathrm{~Hz})$; $6.89(\mathrm{dd}, J=2.0 \& 8.5 \mathrm{~Hz})$ and $7.13(\mathrm{~d}, J=8.5 \mathrm{~Hz})$ and three protons of a terminal double bond at $\delta_{\mathrm{H}}$ $6.63(\mathrm{dd}, J=11.0 \& 17.5 \mathrm{~Hz}), 5.65(\mathrm{~d}, J=17.5 \mathrm{~Hz})$ and $5.13(\mathrm{~d}, J=11.0 \mathrm{~Hz})$. Two sugar units of 4 were showed to be $\beta$-glucopyranose and $\alpha$ rhamnopyranose by revealing of the anomeric signals at $\delta_{\mathrm{H}} 4.75(\mathrm{~d}, J=6.5 \mathrm{~Hz}), \delta_{\mathrm{C}} 104.12$ and $\delta_{\mathrm{H}}$ 4.76 (br s), $\delta_{\mathrm{C}} 102.25$, respectively in the NMR spectra. The linkage of the $\alpha$-rhamnose to the $\beta$ glucose is the same as rutinose, indicating by the HMBC correlation of H-1" $\left(\delta_{\mathrm{H}} 4.76\right)$ to C-6' $\left(\delta_{\mathrm{C}}\right.$ 67.97). The position of rutinose and ethenyl moieties was confirmed by HMBC correlations between $\mathrm{H}-1$ ' $\left(\delta_{\mathrm{H}} 4.75\right)$ and $\mathrm{C}-1\left(\delta_{\mathrm{C}} 146.59\right), \mathrm{H}-7\left(\delta_{\mathrm{H}} 6.63\right)$ and $\mathrm{C}-4$ $\left(\delta_{C} 135.01\right), C-5\left(\delta_{C} 119.52\right)$ and $C-3\left(\delta_{C} 114.37\right)$. The above mentioned analysis led to conclude that 4 is 2-hydroxy-4-ethenylphenyl- $\alpha$-L-rhamnopyranosyl -(1-6)- $\beta$-D-glucopyranoside (brachyanin D) [5]. This compound was isolated for the first time from Stauntonia brachyanthera Hand-Mazz in 2015.

Compound $\mathbf{5}$ obtained as a colorless liquid and gave the pseudo-molecular ion peak at $\mathrm{m} / \mathrm{z} 234$ $[\mathrm{M}+2 \mathrm{H}]^{+}$in the positive ESI-MS spectrum, according to the molecular formula $\mathrm{C}_{9} \mathrm{H}_{12} \mathrm{O}_{7}$. Its NMR data confirmed the presence of one alkenyl moiety signal at $\left.\left(\delta_{\mathrm{H}} 8.02\right) / \delta_{\mathrm{C}} 153.03,142.73\right)$; an oxy-methylen group at $\delta_{\mathrm{H}} 3.86(\mathrm{dd}, J=3.0 \& 12.0$ $\left.\mathrm{Hz}), 3.75(\mathrm{dd}, J=2.5 \& 12.0 \mathrm{~Hz}) / \delta_{\mathrm{C}} 62.29\right)$; five oxy-methine group at $\delta_{\mathrm{H}} 5.71(\mathrm{~d}, \mathrm{~J}=7.5 \mathrm{~Hz}) / \delta_{\mathrm{C}}$ $102.65 ; \delta_{\mathrm{H}} 5.91(\mathrm{~d}, J=4.5 \mathrm{~Hz}) / \delta_{\mathrm{C}} 90.76 ; \delta_{\mathrm{H}} 4.20(\mathrm{t}$, $J=5.0 \mathrm{~Hz}) / \delta_{\mathrm{C}} 75.73 ; \delta_{\mathrm{H}} 4.16(\mathrm{t}, J=5.0 \mathrm{~Hz}) / \delta_{\mathrm{C}} 71.31$ and $\delta_{\mathrm{H}} 4.02(\mathrm{~m}) / \delta_{\mathrm{C}} 86.38$ together with one lactone group at $\delta_{\mathrm{C}} 166.19$. The linkages of two rings were established by HMBC correlations of $\mathrm{H}-4\left(\delta_{\mathrm{H}} 8.02\right)$ to $\mathrm{C}-2\left(\delta_{\mathrm{C}} 166.19\right), \mathrm{C}-5\left(\delta_{\mathrm{C}} 102.65\right)$ and $\mathrm{C}-2{ }^{\prime}\left(\delta_{\mathrm{C}}\right.$ 90.76). The relative stereochemistry of $\mathbf{5}$ was determined by NOESY correlations between $\mathrm{H}$ 4'/H-5', H-5'/H-2' indicating that $\mathrm{H}-2$ ', $\mathrm{H}-4$ ' and $\mathrm{H}-$ 5 ' are on the same side of the molecule. 
VJC, 55(4) 2017

Consequently, the structure of $\mathbf{5}$ was concluded to be cannabiside B by comparison with reported data [6]. It was isolated the first time from Senecio cannabifolius and showed antibacterial activities
Chemical constituents of Chirita drakei Burtt. Part 2.

against Gram-positive bacteria Staphylococcus aureus and Bacillus subtilis.

The ${ }^{1} \mathrm{H}$ and ${ }^{13} \mathrm{C}-\mathrm{NMR}$ data of compounds 1-5 were given in tables 1 and 2 .

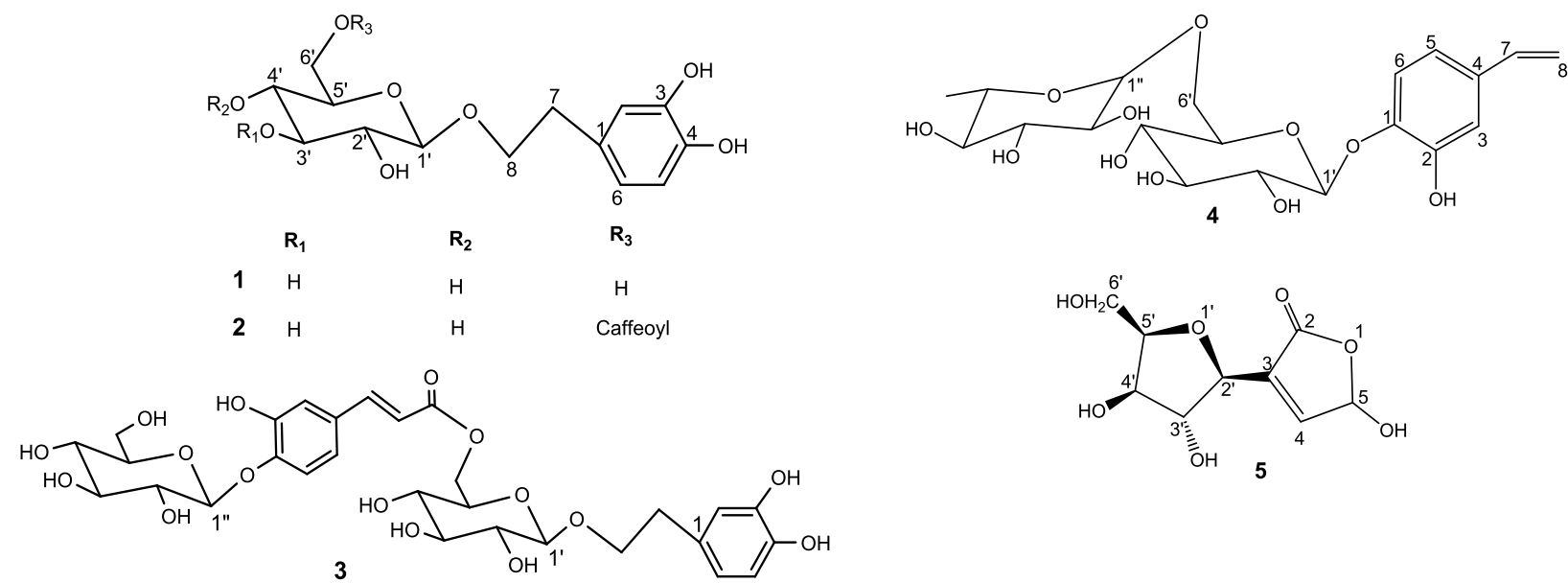

The structure of compounds 1-5

Table 1: ${ }^{1} \mathrm{H}-\mathrm{NMR}$ spectroscopic data for compounds $\mathbf{1 - 5}\left(500 \mathrm{MHz}, \mathrm{CD}_{3} \mathrm{OD}\right)$

\begin{tabular}{|c|c|c|c|c|c|}
\hline Position & 1 & 2 & 3 & 4 & 5 \\
\hline 2 & $6.71 \mathrm{~d}(1.5)$ & $6.69 \mathrm{~d}(2.0)$ & $6.69 \mathrm{~d}(2.0)$ & - & - \\
\hline 3 & - & - & - & $6.97 \mathrm{~d}(2.0)$ & - \\
\hline 4 & - & - & - & - & $8.02 \mathrm{~d}(8.0)$ \\
\hline 5 & $6.69 \mathrm{~d}(8.5)$ & $6.65 \mathrm{~d}(8.0)$ & $6.65 \mathrm{~d}(8.0)$ & $6.89 \mathrm{dd}(2.0 \& 8.5)$ & $5.71 \mathrm{~d}(7.5)$ \\
\hline 6 & $\begin{array}{c}6.57 \mathrm{dd} \\
(1.5 \& 8.5)\end{array}$ & $6.56 \mathrm{dd}(2.0 \& 8.0)$ & $\begin{array}{c}6.55 \mathrm{dd} \\
(2.0 \& 8.0)\end{array}$ & $7.13 \mathrm{~d}(8.5)$ & - \\
\hline 7 & $2.80 \mathrm{~m}$ & $2.81 \mathrm{t}(6.7)$ & $2.81 \mathrm{t}(7.5)$ & $\begin{array}{c}6.63 \mathrm{dd} \\
(11.0 \& 17.5) \\
\end{array}$ & - \\
\hline 8 & $\begin{array}{l}3.72 \mathrm{~m} \\
4.05 \mathrm{~m}\end{array}$ & $\begin{array}{l}3.74 \mathrm{~m} \\
3.99 \mathrm{~m}\end{array}$ & $\begin{array}{l}3.74 \mathrm{~m} \\
3.96 \mathrm{~m}\end{array}$ & $\begin{array}{l}5.65 \mathrm{~d}(17.5) \\
5.13 \mathrm{~d}(11.0) \\
\end{array}$ & - \\
\hline \multicolumn{6}{|c|}{ Glucose-1 } \\
\hline $1 '$ & $4.31 \mathrm{~d}(8.0)$ & $4.35 \mathrm{~d}(8.0)$ & $4.35 \mathrm{~d}(8.0)$ & $4.75 \mathrm{~d}(6.5)$ & - \\
\hline 2 ' & $3.20 \mathrm{t}(8.0)$ & $3.24 \mathrm{t}(8.0)$ & $3.24 \mathrm{t}(8.0)$ & $3.38 \mathrm{~m}$ & $5.91 \mathrm{~d}(4.5)$ \\
\hline 3, & \multirow{3}{*}{$3.28-3.39 \mathrm{~m}$} & \multirow{3}{*}{$3.38 \mathrm{~m}$} & \multirow{3}{*}{$3.32-3.99 \mathrm{~m}$} & $3.47 \mathrm{~m}$ & $4.20 \mathrm{t}(5.0)$ \\
\hline $4 '$ & & & & $3.73 \mathrm{~m}$ & $4.16 \mathrm{t}(5.0)$ \\
\hline 5 & & & & $3.57 \mathrm{~m}$ & $4.02 \mathrm{~m}$ \\
\hline $6^{\prime}$ & $\begin{array}{c}3.68 \mathrm{dd} \\
(5.0 \& 12.0) \\
3.88 \mathrm{dd} \\
(1.5 \& 12.0)\end{array}$ & $\begin{array}{c}4.36 \mathrm{dd} \\
(5.5 \& 11.5) \\
4.51 \mathrm{dd}(2.0 \& \\
11.5)\end{array}$ & $\begin{array}{l}4.34 \mathrm{dd} \\
(5.5 \& 11.5) \\
4.55 \mathrm{dd} \\
(2.0 \& 11.5)\end{array}$ & $\begin{array}{c}4.06 \mathrm{dd} \\
(1.5 \& 11.0) \\
3.64 \mathrm{dd} \\
(6.5 \& 11.0)\end{array}$ & $\begin{array}{c}3.86 \mathrm{dd} \\
(3.0 \& 12.0) \\
3.75 \mathrm{dd} \\
(2.5 \& 12.0)\end{array}$ \\
\hline \multicolumn{6}{|l|}{\begin{tabular}{|l|} 
Caffeoyl \\
\end{tabular}} \\
\hline 2 & - & $7.05 \mathrm{~d}(1.5)$ & $7.11 \mathrm{~d}(2.0)$ & - & - \\
\hline 5 & - & $6.78 \mathrm{~d}(8.0)$ & $7.20 \mathrm{~d}(8.5)$ & - & - \\
\hline 6 & - & $\begin{array}{c}6.91 \mathrm{dd} \\
(1.5 \& 8.0)\end{array}$ & $\begin{array}{c}6.94 \mathrm{dd} \\
(2.0 \& 8.5) \\
\end{array}$ & - & - \\
\hline$\beta$ & - & $6.30 \mathrm{~d}(15.7)$ & & - & - \\
\hline$\gamma$ & - & $7.58 \mathrm{~d}(15.7)$ & & - & - \\
\hline
\end{tabular}




\begin{tabular}{|c|c|c|c|c|c|}
\hline \multicolumn{4}{|c|}{ Glucose-2 } & \multicolumn{2}{|l|}{ Rhamnose } \\
\hline 1" & - & - & $4.88 \mathrm{~d}(7.5)$ & 4.76 br s & - \\
\hline 2" & - & - & \multirow{5}{*}{$3.32-3.99 \mathrm{~m}$} & $3.38 \mathrm{~m}$ & - \\
\hline 3" & - & - & & $3.89 \mathrm{~m}$ & - \\
\hline $4 "$ & - & - & & $3.50 \mathrm{~m}$ & - \\
\hline $5 "$ & - & - & & $\begin{array}{c}3.69 \mathrm{dd} \\
(6.5 \& 9.5)\end{array}$ & - \\
\hline 6" & & & & $1.25 \mathrm{~d}(6.5)$ & - \\
\hline
\end{tabular}

Table 2: ${ }^{13} \mathrm{C}-\mathrm{NMR}$ spectroscopic data for compounds $\mathbf{1 - 5}\left(125 \mathrm{MHz}, \mathrm{CD}_{3} \mathrm{OD}\right)$

\begin{tabular}{|c|c|c|c|c|c|}
\hline No & 1 & 2 & 3 & 4 & 5 \\
\hline 1 & $131.55 \mathrm{C}$ & $131.42 \mathrm{C}$ & $131.34 \mathrm{C}$ & $146.59 \mathrm{C}$ & - \\
\hline 2 & $117.13 \mathrm{CH}$ & $117.12 \mathrm{CH}$ & $117.08 \mathrm{CH}$ & $148.31 \mathrm{C}$ & $166.19 \mathrm{C}$ \\
\hline 3 & $146.11 \mathrm{C}$ & $146.06 \mathrm{C}$ & $146.16 \mathrm{C}$ & $114.37 \mathrm{CH}$ & $153.03 \mathrm{C}$ \\
\hline 4 & $144.64 \mathrm{C}$ & $144.57 \mathrm{C}$ & $144.67 \mathrm{C}$ & $135.01 \mathrm{C}$ & $142.73 \mathrm{CH}$ \\
\hline 5 & $116.33 \mathrm{CH}$ & $116.42 \mathrm{CH}$ & $116.38 \mathrm{CH}$ & $119.52 \mathrm{CH}$ & $102.65 \mathrm{CH}$ \\
\hline 6 & $121.27 \mathrm{CH}$ & $121.30 \mathrm{CH}$ & $121.24 \mathrm{CH}$ & $118.55 \mathrm{CH}$ & - \\
\hline 7 & $36.55 \mathrm{CH}_{2}$ & $36.62 \mathrm{CH}_{2}$ & $36.74 \mathrm{CH}_{2}$ & $137.65 \mathrm{CH}$ & - \\
\hline 8 & $72.09 \mathrm{CH}_{2}$ & $72.38 \mathrm{CH}_{2}$ & $72.45 \mathrm{CH}_{2}$ & $112.84 \mathrm{CH}_{2}$ & - \\
\hline \multicolumn{6}{|c|}{ Glucose-1 } \\
\hline $1 '$ & $104.35 \mathrm{CH}$ & $104.47 \mathrm{CH}$ & $104.57 \mathrm{CH}$ & $104.12 \mathrm{CH}$ & - \\
\hline 2 ' & $75.11 \mathrm{CH}$ & $75.01 \mathrm{CH}$ & $75.08 \mathrm{CH}$ & $74.06 \mathrm{CH}$ & $90.76 \mathrm{CH}$ \\
\hline 3 ' & $77.92 \mathrm{CH}$ & $77.87 \mathrm{CH}$ & $77.97 \mathrm{CH}$ & $77.63 \mathrm{CH}$ & $75.73 \mathrm{CH}$ \\
\hline $4 '$ & $71,62 \mathrm{CH}$ & $71.70 \mathrm{CH}$ & $71.85 \mathrm{CH}$ & $72.43 \mathrm{CH}$ & $71.31 \mathrm{CH}$ \\
\hline 5, & $78.07 \mathrm{CH}$ & $75.36 \mathrm{CH}$ & $75.42 \mathrm{CH}$ & $77.10 \mathrm{CH}$ & $86.38 \mathrm{CH}$ \\
\hline $6^{\prime}$ & $62.72 \mathrm{CH}_{2}$ & $64.64 \mathrm{CH}_{2}$ & $64.81 \mathrm{CH}_{2}$ & $67.97 \mathrm{CH}_{2}$ & $62.29 \mathrm{CH}_{2}$ \\
\hline \multicolumn{6}{|l|}{ Caffeoyl } \\
\hline 1 & - & $127.65 \mathrm{C}$ & $130.99 \mathrm{C}$ & - & - \\
\hline 2 & - & $115.10 \mathrm{CH}$ & $115.80 \mathrm{CH}$ & - & - \\
\hline 3 & - & $146.73 \mathrm{C}$ & $148.49 \mathrm{C}$ & - & - \\
\hline 4 & - & $149.59 \mathrm{C}$ & $148.92 \mathrm{C}$ & - & - \\
\hline 5 & - & $116.58 \mathrm{CH}$ & $118.11 \mathrm{CH}$ & - & - \\
\hline 6 & - & $123.20 \mathrm{CH}$ & $122.45 \mathrm{CH}$ & - & - \\
\hline$\alpha$ & - & $169.29 \mathrm{C}$ & $168.69 \mathrm{C}$ & - & - \\
\hline$\beta$ & - & $114.80 \mathrm{CH}$ & $117.08 \mathrm{CH}$ & - & - \\
\hline$\gamma$ & - & $147.29 \mathrm{CH}$ & $146.37 \mathrm{CH}$ & - & - \\
\hline \multicolumn{4}{|c|}{ Glucose-2 } & \multicolumn{2}{|l|}{ Rhamnose } \\
\hline $1 "$ & - & - & $103.45 \mathrm{CH}$ & $102.25 \mathrm{CH}$ & - \\
\hline 2" & - & - & $74.81 \mathrm{CH}$ & $71.60 \mathrm{CH}$ & - \\
\hline 3" & - & - & $77.56 \mathrm{CH}$ & $72.19 \mathrm{CH}$ & - \\
\hline 4" & - & - & $71.35 \mathrm{CH}$ & $74.88 \mathrm{CH}$ & - \\
\hline $5 "$ & - & - & $78.36 \mathrm{CH}$ & $69.89 \mathrm{CH}$ & - \\
\hline 6" & - & - & $62.47 \mathrm{CH}_{2}$ & $17.95 \mathrm{CH}_{3}$ & - \\
\hline
\end{tabular}

Acknowledgments. We would like to thank the National Foundation for Science and Technology
Development of Vietnam (NAFOSTED) for financial support (Reference code 104.01.2013.59). 


\section{REFERENCES}

1. Nguyen Thi Hoang Anh, Nguyen Van Tuan, Tran Duc Quan, Dao Duc Thien, Nguyen Thanh Tam, Trinh Thi Thuy, Tran Van Sung. Chemical constituents of Chirita drakei Burtt collected in $\mathrm{Ha}$ long Bay, Quang ninh province, Viet Nam. I. Compounds isolated from n-hexane and ethyl acetate extracts, Journal of Chemistry, 55(2), 203-207 (2017).

2. Hiroko Shimomur, Yutaka Sashida, Tokuo Adachi. Phenolic glucosides from Prunus grayana Phytochemistry, 26(1), 249 (1987).

3. Jan Schlauer, Jaromir Budzianowski, Krystyna Kukulczanka, Lidia Ratajczak. Acteoside and related phenylethanoid glycosides in Byblis liniflora Salisb.
Plants propagated in vitro and its systematic significance, Acta Societatis Botanicorum Poloniae, 73, 9-15 (2004).

4. Soren Damtoft, Soren Rosendal Jensen. Three phenylethanoid glucosides of unusual structure from Chirita sinensis (Gesneriaceae), Phytochemistry, 37(2), 441-443 (1994).

5. Jing Zhao, Jia Guo, Yi Zhang, Dali Meng, Zhou Sha. Chemical constituents from the roots and stems of Stauntonia brachyanthera Hand-Mazz and their bioactivities. Journal of Functional Foods, 14, 374383 (2015).

6. Bin Wu, Wen Hui Lin, Hui Yuan Gao, Lu Zheng, Li Jun Wu, Chul Sa Kim. Four New Antibacterial Constituents from Senecio cannabifolius. Pharmaceutical Biology, 44, 440-444 (2006).

\author{
Corresponding author: Nguyen Thi Hoang Anh \\ Institute of Chemistry \\ Vietnam Academy of Science and Technology \\ No. 18, Hoang Quoc Viet Road, Cau Giay Dist., Hanoi \\ E-mail: hoanganhvhh@gmail.com.
}

